\title{
BMJ Open Qualitative evaluation of a deferred consent process in paediatric emergency research: a PREDICT study
}

\author{
Jeremy Furyk, ${ }^{1,2,3,4}$ Kristin McBain-Rigg, ${ }^{1}$ Kerrianne Watt, ${ }^{1}$ Theophilus I Emeto, \\ Richard C Franklin, ${ }^{1}$ Donna Franklin, ${ }^{5,6}$ Andreas Schibler, ${ }^{5,6}$ Stuart R Dalziel, ${ }^{7,8}$ \\ Franz E Babl, ${ }^{3,9}$ Catherine Wilson, ${ }^{3}$ Natalie Phillips, ${ }^{10}$ Robin Ray, ${ }^{4}$ on behalf of \\ PREDICT ${ }^{11}$
}

\begin{abstract}
To cite: Furyk J, McBainRigg K, Watt K, et al. Qualitative evaluation of a deferred consent process in paediatric emergency research: a PREDICT study. BMJ Open 2017;7:e018562. doi:10.1136/ bmjopen-2017-018562

- Prepublication history and additional material for this paper are available online. To view these files, please visit the journal online (http://dx.doi. org/10.1136/bmjopen-2017018562).
\end{abstract}

Received 8 July 2017 Revised 2 September 2017 Accepted 18 September 2017

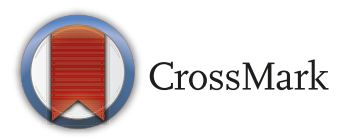

For numbered affiliations see end of article.

Correspondence to

Dr Jeremy Furyk;

jeremy.furyk@health.qld.gov.au

\section{ABSTRACT}

Background A challenge of conducting research in critically ill children is that the therapeutic window for the intervention may be too short to seek informed consent prior to enrolment. In specific circumstances, most international ethical guidelines allow for children to be enrolled in research with informed consent obtained later, termed deferred consent (DC) or retrospective consent. There is a paucity of data on the attitudes of parents to this method of enrolment in paediatric emergency research. Objectives To explore the attitudes of parents to the concept of DC and to expand the knowledge of the limitations to informed consent and DC in these situations. Method Children presenting with uncomplicated febrile seizures or bronchiolitis were identified from three separate hospital emergency department databases. Parents were invited to participate in a semistructured telephone interview exploring themes of limitations of prospective informed consent, acceptability of the DC process and the most appropriate time to seek DC. Transcripts underwent inductive thematic analysis with intercoder agreement, using Nvivo 11 software.

Results A total of 39 interviews were conducted. Participants comprehended the limitations of informed consent under emergency circumstances and were generally supportive of DC. However, they frequently confused concepts of clinical care and research, and support for participation was commonly linked to their belief of personal benefit.

Conclusion Participants acknowledged the requirement for alternatives to prospective informed consent in emergency research, and were supportive of the concept of DC. Our results suggest that current research practice seems to align with community expectations.

\section{BACKGROUND}

Conducting clinical trials with critically ill children is frequently associated with ethical dilemma. The therapeutic window for many interventions is too short to seek informed consent, and parents may be unavailable or lack capacity to provide adequately informed consent when their child is critically ill. ${ }^{1}$ Yet critically ill children deserve high-quality care

\section{Strengths and limitations of this study}

- The study addresses the important question of parental attitudes, perceptions and acceptability of deferred consent in paediatric emergency research.

- Qualitative methodology used is well suited to address this question.

- Participants had recent experience in emergency departments, and could contextualise the feelings of anxiety and vulnerability frequently associated with such visits.

- Participants were not involved in any clinical research; therefore, responses are hypothetical.

based on robust evidence of benefit, requiring clinical trials. It is generally not possible to predict in advance which children may be eligible for research in emergency settings, a limitation that makes prior consent unhelpful in most circumstances. To allow robust evidence to be generated, provisions for waiver, or exception to prospective informed consent, in certain narrow circumstances is incorporated into most international ethical guidelines for medical research. ${ }^{2-6}$

The process of seeking consent from a participant, or their proxy, at a time point after an experimental intervention is often termed deferred consent (DC), delayed or retrospective consent. When DC is provided, the participant continues in the trial, and their data are retained for analysis. When DC is not provided, the participant and their prior data are withdrawn from the trial and the analysis. The process of DC, while increasingly common, has a number of ethical dilemmas. ${ }^{7-9}$ Parents do not get the opportunity to refuse the intervention as it has already been instituted by the time consent is sought; consequently, the term 'deferred consent' may be considered misleading, and consent for continued participation and for consent 
to use data might be preferable. Opponents argue that such a process violates the autonomy of patients or parents; however, equally important is the argument that vulnerable populations should not be denied justice and the opportunity to participate in research.

While there is some limited data describing the attitudes, perceptions and the acceptability of DC and other alternatives to prospective informed consent in parents of critically unwell children internationally, ${ }^{6710}$ there are no data available in the Australian context. The objective of this study was to explore and describe the experiences and attitudes of parents of children attending emergency departments (EDs) for acute conditions in relation to participation in research, when prospective informed consent is not possible. This knowledge is vital to help inform the design of future trials that maintain the trust of the community and ensure research adheres to community expectations.

\section{METHODS}

We used a modified grounded theory methodology to describe and explore the phenomenon of parental attitudes to DC in parents of children presenting to EDs for emergency care. ${ }^{11-13}$ The study was reported according to the consolidated criteria for reporting qualitative research statement on qualitative research. ${ }^{14}$

\section{Conceptual perspective}

This study was conceived and developed from the experiences of paediatric emergency physicians. Time-critical and stressful situations impede obtaining meaningful prospective informed consent in both clinical and research contexts. Within medicine, there exists a paradoxical acceptance of using unproven interventions outside of a research protocol without recriminations and prohibitive scrutiny, and of using either proven or unproven interventions without prospective informed consent in true emergency life-threatening situations. If the same interventions are provided as part of research, there is increased regulatory oversight whether collecting deidentified data within negligible risk, observational research or collecting data as part of a randomised controlled trial, the gold standard of robust evidence. Underlying this paradox is the strong belief that emergency research is vital, that interventions used in EDs should be evidence based and that researchers need to engage the general public to ensure that research practices are within acceptable community standards.

\section{Setting}

Data were collected in three Australian EDs: two tertiary urban paediatric facilities and one regional referral, mixed adult and paediatric centre. All are members of the Paediatric Research in Emergency Departments International Collaborative (PREDICT). At the time of the study, there were two PREDICT clinical trials in progress: The Convulsive Status Epilepticus Paediatric
Trial (ConSEPT), an evaluation of levetiracetam versus phenytoin for the second-line management of convulsive status epilepticus ${ }^{15}$; and the high-flow nasal cannula treatment for viral bronchiolitis, a Randomised Controlled Trial-Paediatric Acute Respiratory Intervention Studies (PARIS) trial, which compared nasal high-flow therapy versus standard oxygen therapy in the management of bronchiolitis in infants and the need for escalation including higher level of care or intensive care. ${ }^{16}$ The majority of participants in these studies are previously healthy children, with little or no contact with emergency medical services. In both circumstances, researchers and ethics committees determined that obtaining prospective informed consent would not be possible and a DC process was approved.

\section{Participants}

Using purposive sampling parents of children presenting with simple febrile seizures (non-status epilepticus) and uncomplicated bronchiolitis (not requiring admission) was identified from participating ED databases. Children in this study were not eligible for the two clinical trials described, although presented with milder forms of the same acute presentations. This was to replicate the contexts of the two studies, so that parents could contextualise the proposed research in light of their experiences.

\section{Consent}

Participants were contacted via mail up to 3 months following presentation to hospital to explain the study, with participant information and consent forms, allowing them to 'opt out' of the study (via return mail or email). Participants who opted out were not contacted further. Those who did not opt-out were contacted via telephone and again given the opportunity to decline participation. Those who consented nominated a suitable time for telephone interviewing. At interview, verbal consent was obtained and digitally recorded.

\section{Data collection methods}

Semistructured interviews were guided by a schedule of topics generated from literature and input from ConSEPT and PARIS Bronchiolitis High Flow investigators (online supplementary appendix 1). Open-ended questions encouraged participants to explore other topics and concepts. General topics included various approaches to consent in emergency medicine research, parental understanding of these research processes and decision-making, trial design and acceptability of DC as well as issues of DC in the event of poor outcome or child death. We used an iterative process, where the schedule was refined during the process of data collection and analysis.

Digitally recorded telephone interviews were conducted from March to December 2016 by a trained researcher (KM). Data collection and recruitment continued until no new themes or information was forthcoming from the data indicating that saturation had been achieved. 


\section{Data analysis}

Inductive thematic data analysis followed a modified grounded theory approach, conducted iteratively throughout the study in conjunction with ongoing data collection. Interview recordings were deidentified and transcribed verbatim, and transcripts and audio imported into data management software. All analysis was supported using the qualitative software programme NVivo for Mac (QSR International Pty Ltd V.11, 2016). An initial open-coding structure was developed and was continually refined and clarified as data collection and analysis continued alongside refinements of the interview schedule. Through axial coding, emerging themes were produced through repeated reading and constant comparison between transcripts. Memo writing clarified ideas about the data and concepts regarding parental attitudes as patterns were identified. ${ }^{17}$ This was done contemporaneously with interviews to allow refinement and test any new topics raised by participants that were of relevance to the study. At the completion of all interviews, the text was re-examined using the identified themes and coded accordingly. Audio data were examined with attention to intonation and to gain clarity of issues. A process of intercoder agreement was used to ensure the trustworthiness of the thematic analysis process, and the data further triangulated through discussion of themes in reference to literature on the topic.

\section{RESULTS}

Thirty-nine interviews were conducted over 9 months. Demographic details are presented in table 1. Participants were predominantly female $(85 \%)$, identified only as 'Australian' with no religion or Christianity, were well educated and with half $(54 \%)$ having a household income in excess of $\$ A 100000$.

Without exception, participants were supportive of medical research and research in emergency medicine. Themes arising from the data with regards to DC were positive and negative attitudes to DC; with reasoning behind attitudes categorised as patient/parental factors, trial design and research factors, process factors and specific issues.

\section{Attitude to DC process}

There was general, but not universal, support for research in emergency settings with DC. Demographic details did not seem to influence positive or negative attitude towards DC, neither did the condition at presentation (bronchiolitis or febrile seizure). Participants discussed several barriers to obtaining meaningful prospective informed consent such as the time-critical element of emergency research, the highly emotive environment contributing to impaired decision-making capacity:

I think in an emergency situation, you know, whatever has to happen has to happen
Table 1 Participant demographics details

n (\%)

\begin{tabular}{lr}
\hline Hospital & \\
TTH & $20(51)$ \\
RCH & $10(26)$ \\
LCCH & $9(23)$ \\
& Total $39(100)$
\end{tabular}

Presentation

$\begin{array}{lr}\text { Bronchiolitis } & 22(66) \\ \text { Febrile seizure } & 17(44) \\ \text { Age (years) } & \\ 18-34 & 18(46) \\ 35-44 & 17(44) \\ 45+ & 4(10) \\ \text { Sex } & \end{array}$

Female

$33(85)$

Ethnicity

Australian

$24(61)$

ATSI

1 (3)

Asian

4 (10)

Other

8 (21)

Not specified

2 (5)

Religion

None

$18(46)$

Christian

13 (33)

Buddhism

1 (3)

Islam

1 (3)

Jehovah's witness

1 (3)

Other/not identified

$5(13)$

Education

$\begin{array}{lr}\text { Did not complete year } 12 & 4(10) \\ \text { Completed year } 12 \text { only } & 5(13) \\ \text { Postschool/non-university } & 11(28) \\ \text { Undergraduate university } & 15(38) \\ \text { Postgraduate university } & 4(10) \\ \text { Annual household income } & \\ \text { Less than \$A50K } & 6(15) \\ \text { \$A50-\$A100K } & 8(21) \\ \text { More than \$A100K } & 21(54) \\ \text { Unsure } & 4(10)\end{array}$

ATSI aboriginal and/or Torres Straight Islander, $\$$ are Australian Dollars (AUD).TTH, The Townsville Hospital; GCUH, Gold Coast University Hospital; LCCH, Lady Cilento Children's Hospital; $\mathrm{RCH}$, Royal Children's Hospital.

I wouldn't want doctors to delay what they needed to do, if it would possibly affect my kid even more by coming out and making sure what I had to know, you know read all this and read all that, sign all this and sign all that, I just want them to do what they need to do 
Very few participants demonstrated clear negative attitude towards the concept of DC, stating "control had been taken away'.

I don't think asking for consent later would be appropriate

Consent should always be asked before anything, not after anything

Some participants qualified comments suggesting that being 'updated' or 'kept in the loop' was important and influenced support for the concept of DC. Some could see both sides without making a definitive response either way, and indicated a preference for prior consent if at all possible.

So I can understand that sometimes it would be better in emergency just to do what needs to be done even if, especially if it was better for the child, but at the same time I... if time permits I would rather be asked or be informed in advance

\section{Patient/parental factors}

\section{Emotional state}

The majority of parents did not feel that meaningful informed consent was possible in circumstances such as attending the ED with their unwell child. The major barrier identified was their emotional state at this time, variously described as 'anxious', 'freaking out' or 'a state of shock'. Parents indicated they would not have been in the 'right frame of mind' to consider research decisions, with their focus on ensuring the child was being looked after.

when you are in an emergency situation... you're not really taking in everything they're saying anyway

I think when you are in that situation where you are so stressed, it would be extremely difficult for you to read any document or to have someone explain anything to you and for you to actually be able to go through it the way you would when you are not stressed

Those parents who felt able to make meaningful decisions at the time of ED presentation had children who tended to be improving or stable in ED. The emotional burden and ability to process information was perceived as a very personal experience; some participants suggested that their partners would have different opinions and responded differently in the same situation. One participant suggested previous work experiences were a factor contributing to decision-making ability under stressful circumstances.

my partner may not [be capable of decision making], she might be so emotionally affected that, she'd waste time trying to understand

mothers they just stress a little bit more. I think I would have been fine in that situation

in that scenario, I probably would have [been able to make an informed decision about research participation]... only cause I, like I said I am probably used to handling stressful situations [at work experiences]

\section{Preconceptions}

Regardless of the difficulty in interpreting information at certain times, if approached to participate in research under those circumstances, some implied they would be likely to respond in a predetermined way, irrespective of specific details.

I think I am always willing to help with research and I probably would have said yes straight away

I was pretty upset at the time already, and then if you think about a study you would be like, no, no, no just try the normal thing.

\section{Trust in medical teams}

Generally positive attitudes to research with DC were accompanied by the theme of trust in medical teams. Parents generally expressed confidence that treating clinicians had the required expertise to make the best decisions for their child and had the best interest of the child in mind.

you guys are the professionals and if it is endorsed by the Hospital then I would be happy, honestly, like I'm not a doctor and I will never try to override what a doctor is saying and wants to do in doing their job

I wouldn't bat an eyelid if we had gone in there and you [the doctor] said look this is what we are doing

Research understanding and perceived personal benefit

Some comments suggested participants' demonstrated only a limited understanding of the research process, and often had the perception of personal benefit from research participation. Support for research with DC was occasionally conditional on such benefit.

It would have been [acceptability of research with DC] as long as it was in the best interest of my child and was going to get him better

If it was going to save his life, then yes [would be acceptable]

\section{Trial design and research factors}

Clinical severity and emergency situations

The 'critical' or 'life-threatening' nature of the condition, as well as the time-critical nature of the proposed intervention influenced attitude to research with DC. Most often, participants indicated a greater acceptability of a DC process in these circumstances.

I think if their child was critically ill and there was... no time for a parent to process all that information, then I think that a parent will understand

if it was life threatening I would say please do whatever you have to do, but if it is not necessarily life threatening and then there's going to be unknown consequences...I would like to be able to make that choice myself 
Potential harm

The potential 'risk' or 'unknown consequences' associated with research was another factor that concerned parents. Whether the intervention was commonly used or equivalent to 'standard care' was important to some.

I would suppose in that case it would be [acceptable], as long as the proposed method is going to be just as safe as the regular way

you don't want to ever feel like you're putting your child at risk.

\section{Complexity}

The complexity of the proposed intervention also influenced the acceptability of the DC process. For example, when the intervention was considered to be uncomplicated, informed consent might be possible in some form.

if the research was reasonably straightforward, I think it's okay, I think you could still be stressed and you know sort of consent

\section{Process factors}

\section{Ethics committee approval}

Participants were mostly comfortable with the hospital ethics/institutional review board review procedure, and considered that these processes protected individuals' rights and well-being when participating in research. A minority acknowledged the limitations of the process.

you guys are the professionals and if it is endorsed by the Hospital then I would be happy

an ethics committee is neutral and they know the guidelines to go by and what lines not to cross and all that sort of thing, so yeah, and that to me is fine

I mean committees aren't perfectly made up of people and everybody, people have their faults, their flaws and agendas

\section{Community consultation}

The concept of community consultation was less well supported. Some responses indicated that the process may not add value, and that the 'community' chosen may not necessarily represent their personal opinions, beliefs and values.

as long as they are asking the right focus groups..., 'cause different people have an opinion who shouldn't have an opinion'

but everyone doesn't have the same opinion as me

\section{Legal issues and paperwork}

Informed consent was often considered synonymous with the act of completing paperwork rather than the exchange of information. Experiences of consent processes in other circumstances, such as for routine or emergency clinical care, contributed to this notion. Some viewed the process solely as a legal issue required to 'protect both parties'.

on the night you might have signed the consent which may not mean anything because you know you are all over the place already and you just sign any paperwork that they put in front of you

$\mathrm{j}$ ust scribble a signature on a piece of paper if you really need to

\section{The DC procedure}

The most appropriate time to approach parents for consent was considered to be 'as soon as possible' but to wait until the situation had 'calmed down' or 'stabilised', for both child and parent. Parents valued being kept informed or 'in the loop' about decisions being made both in research and in clinical care. The benefit of having a dedicated support person available during the process was also mentioned.

In the situation where I was in, probably no, [I wouldn't have consented to participate in research] [be]cause I was there by myself, if there was somebody else, probably yes.

the ideal situation [is] usually [to] have several doctors that are able to, one is able to start on what's going on... another doctor is able to come and explain what is happening

I think that [being enrolled in research without prior consent] would make me feel pretty uncomfortable if I wasn't being told what was going on

\section{Specific issues}

Child death

There was considerable variation in responses regarding whether consent should be sought, or data included without consent (waiver of consent), when children died during a research study prior to obtaining consent from families. Some participants felt strongly that consent should be sought, citing respect for the family's right to know details of the circumstances. However, other participants expressed concern that informing the family would not benefit them, and may potentially cause stress and anxiety.

\section{Definitely have to ask}

there might be unfortunate outcomes but you have still got to go and seek consent

The complexity of the issue was highlighted by contrasting views advocating inclusion of data without seeking consent.

I mean if you are just looking at pure statistic numbers, and nothing more... I think just use the data

you're not putting through parents anything on top of what they have already been through

I would say I wouldn't even bother telling them, honestly

Some parents brought up the issue of potential bias in such cases. The issue of confidentiality was more important when discussing child death than in other circumstances.

But if the parents said no it wasn't included well then that stuffs up things doesn't it? 
if you didn't count the children that passed, the treatment, it wouldn't be too statistical

If, if someone dies, and that's not used in the study, that's precious information lost.

One reason given for seeking consent was demonstration of the concept of beneficence. Participants felt that knowledge and skill gained when participating in research may result in contribution to the 'greater good' or something positive coming from the tragic situation, might be of comfort to grieving families.

\section{I would want to know that the data from what would have happened with my child might help another child}

Variability in responses extended to the best time to seek DC in such situations. While most agreed that this should be performed after a suitable period of grief was allowed, this varied from 'a few hours', to 'weeks', 'months' or 'case by case'. Most felt that contact should occur within weeks of the child's passing, and that it should be in a face-to-face context.

Don't send a letter, it's got to be face to face, It's got to be personal'

\section{DISCUSSION}

Our study of parents of infants and children attending EDs with bronchiolitis or febrile seizures found a generally positive attitude to DC in emergency research involving time-critical and life-threatening situations. Our results are broadly consistent with the international qualitative and quantitative research in the field. ${ }^{6} 710$ 18-25 Surveys in various populations including scenarios of adult trials found the majority of respondents would be willing to participate in research without informed consent, ${ }^{18-20} 222325$ which seems to be consistent in paediatric studies. ${ }^{67102124}$

Participants in our study acknowledged barriers to obtaining valid or meaningful informed consent in emergent circumstances due to their emotional state and limited time available. This is situational incapacity and is congruent with previous studies. ${ }^{6} 91024$ A United Kingdom group examined DC in a hypothetical trial similar to one of the scenarios presented in our study. ${ }^{10}$ Parents described that capacity to provide informed consent in such circumstances was likely to be impaired, and they trusted practitioners to make research-related decisions. ${ }^{10}$ Parents reported DC to be more acceptable if both treatment options represented 'standard care' or were 'low risk', and less acceptable if higher risk interventions were involved. Also influencing the acceptability of DC was the 'critical nature of the illness' and the therapeutic window, or how urgently the intervention needed to be administered. It is reassuring that these comments reflect existing guidance ${ }^{426}$ on research without consent, which implies that guidance is in line with community expectations.
Ideas of keeping parents informed or 'in the loop' or of limited consent expressed as 'sort of consent' were raised during interviews. Many participants expressed that informed consent was preferred if possible or if time permits'. A staged consent process was used in a large paediatric critical care trial with mixed results. ${ }^{827}$ The Fluid Expansion as Supportive Therapy (FEAST) trial which explored the effect of intravenous fluids boluses in critically unwell children in Africa sought the 'assent' of parents prior to enrolment of children into the trial according to a predetermined script. ${ }^{827}$ This was followed by formal written informed consent to continue in the trial and use of data. Advantages are that participants are aware of the research and have the opportunity to 'opt out' or decline participation, although this decision may not be based on a balanced assessment of the risks and benefits of participation. An opportunity to decline participation may have appealed to respondents in our study who had preconceptions about clinical trial participation, and may be perceived as respecting individuals' beliefs and values. In the FEAST trial, preconceptions were thought to contribute to automatic refusing or agreeing based on previous negative or positive experiences. ${ }^{9}$ The opportunity to decline participation has also been identified as important in other studies. ${ }^{24}$ While a qualitative evaluation of the FEAST trial consent process highlighted some limitations, ${ }^{9}$ it may be worth exploring further in other settings.

Some participant responses in our study suggested that research understanding might be suboptimal, particularly with regard to the perception of personal benefit. The lack of distinction between clinical care and research has been labelled 'therapeutic misconception', ${ }^{28} 29$ and is not unique to research in emergency settings. Estimates indicate that this is an issue up to $70 \%$ of the time in a variety of research settings. ${ }^{28}$ The validity of consent under these circumstances is questionable. This concept was at times enmeshed with the theme of trust in medical teams to make research decisions, which seemed to contribute significantly to respondents' positive attitudes to participation in research with DC which has been previously reported. ${ }^{10}$ While it is pleasing to think that the general public has confidence in the medical profession, and many respondents clearly understood the experimental nature of a clinical trial, in the setting of a research project the preposition that medical teams 'know what is best', or act in the patients 'best interests', is perhaps contrary to the concept of equipoise that justifies any ethical research. It may be that participants were expressing the related concept that they were confident that doctors would not be exposing patients to additional risk, but this is speculation and should be explored further in future studies. Parents were most comfortable with comparisons of two equally acceptable alternative interventions, without evidence of superiority and the concept of low or negligible 'incremental' risk.

In our cohort, the least consistent responses were found in discussions about DC in the event of child death 
during a trial. Opinions were divergent on whether data should be used automatically, or consent always sought. In a postal survey in the UK, two-thirds of bereaved families anticipated wanting to be informed of trial participation at some time. ${ }^{7}$ The deeply personal and difficult nature of this scenario may mean that generalisations are not possible. Researchers may need to consider that a 'one size fits all' approach is not appropriate, and a tailored approach taking into account patients preferences, values and beliefs is required. Implementing such an approach may require special skills. ${ }^{10}$ Astute participants raised the potential for bias when data on bad outcomes was not collected. This has been shown to be a significant problem in the recent UK CATheter infections in CHildren $(\mathrm{CATCH})$ trial, evaluating three different central line devices in elective surgery with prospective consent, and critically unwell acute presentations without prospective informed consent later approached for DC to use data collected. ${ }^{30}$ The trial had a high mortality overall; however, the likelihood of being approached for consent was different according to outcome, disproportionately excluding children who died, and only $72 \%$ of patients randomised on emergency basis had DC obtained. The authors conclude that researchers and ethics committees need to balance the additional burden of seeking consent with the potential for bias by excluding such cases ${ }^{30}$ With our data suggesting divided opinion, this is an important issue for ethics committees, with the consideration of using a waiver of informed consent for primary outcome data.

Community consultation and public disclosure is a requirement in the United States for research without consent. The process has been criticised as vague and poorly defined, with identification of an appropriate 'community' to seek views for research in emergency settings being problematic. ${ }^{18} 31$ This process was not viewed favourably by participants in our study, with parents aware that individuals participating in community consultation might not reflect their views.

Our study had a number of limitations. The population sampled was parents of infants and children attending the ED with bronchiolitis and febrile convulsions. This population was chosen to contextualise two concurrent randomised trials using DC in critically ill children. Due to resource and logistical issues, interviews were conducted up to 3 months after the presentation, which may have lead to some recall bias. Some of the included parents reported not being distressed or anxious when their children presented to the ED, and consequently they may not have been able to provide insights reflecting a true emergency situation when children present with more severe disease. However, the vast majority of participants reported distress when presenting to the ED and were mindful of the emotional impact of this situation. Second, our study was conducted in an Australian population, and may not be representative of other settings. The study population was relatively wealthy and well educated; therefore, caution is advised in transferring results to other settings. However, we purposively sought to include parents from two state capital cities and a metropolitan centre, testing the relevance of findings in different settings. Third, fewer fathers (than mothers) participated limiting this important perspective. However, this is reflective of ED presentations of children in general and the population from whom consent is likely to be obtained. Finally, parents in our study did not have direct experience of this consent process or clinical trials; therefore, their responses, although informed by recent experiences, may not reflect actual responses if exposed to this process.

In conclusion, we found parents attending EDs with their children to be broadly supportive of DC in paediatric emergency research, and aware of the limitations of prospective informed consent in emergency situations. Concerns of parents are broadly reflective of existing guidance on research in these circumstances, suggesting that current research practice seems to align with community expectations. DC in cases of child death was a difficult and contentious issue, which needs careful consideration by researchers and ethics committees when planning future clinical trials.

\section{Author affiliations}

${ }^{1}$ College of Public Health, Medical and Veterinary Sciences, James Cook University, Townsville, Queensland, Australia

${ }^{2}$ Emergency Department, The Townsville Hospital, Townsville, Queensland, Australia ${ }^{3}$ Murdoch Childrens Research Institute, Melbourne, Victoria, Australia

${ }^{4}$ College of Medicine and Dentistry, James Cook University, Townsville, Queensland, Australia

${ }^{5}$ Paediatric Critical Care Research Group, Lady Cilento Children's Hospital, Brisbane, Queensland, Australia

${ }^{6}$ Mater Research Institute - The University of Queensland, Brisbane, Australia

${ }^{7}$ Starship Children's Hospital, Auckland, New Zealand

${ }^{8}$ The University of Auckland, Auckland, New Zealand

${ }^{9}$ Emergency Department, Royal Childrens Hospital, Parkville, Victoria, Australia

${ }^{10}$ Emergency Department, Lady Cilento Children's Hospital, Brisbane, Queensland, Australia

${ }^{11}$ Paediatric Research in Emergency Departments International Collaborative

Contributors JF, KMR and RAR contributed to the conception and development of the study, project management, reporting and publication. JF obtained funding. $\mathrm{JF}, \mathrm{KM}-\mathrm{R}, \mathrm{DF}, \mathrm{AS}, \mathrm{CW}$, FEB and SRD developed the interview schedule. KM-R, CW, $\mathrm{FEB}, \mathrm{NP}, \mathrm{DF}$ and AS participated in participant recruitment and data collection. $\mathrm{KM}-\mathrm{R}$ performed all interviews. JF, KM-R and RAR developed and refined the coding framework, and performed the data analysis. JF prepared the first draft of the manuscript, and all authors contributed to revisions and had full access to data. JF takes responsibility for the paper as a whole.

Funding The study was part supported by a grant by the Townsville Hospital, Study, Education and Research Trust Account. Th PI (JF) is supported by a research scholarship from The Emergency Medicine Foundation EMRS-51R25-2016.

Competing interests None declared.

Ethics approval The study was approved by the Townville Hospital and Health Service, Human Research Ethics Committee (HREC/15/QTHS/206), the Royal Children's Hospital Human Research Ethics Committee (HREC: 35279A), James Cook University Human Research Ethics Committee (HREC: H6468) and Children's Health Queensland Human Research Ethics Committee (HREC/16/QRCH/137).

Provenance and peer review Not commissioned; externally peer reviewed.

Data sharing statement Deidentified data can be made available on request by corresponding author.

Open Access This is an Open Access article distributed in accordance with the Creative Commons Attribution Non Commercial (CC BY-NC 4.0) license, which 
permits others to distribute, remix, adapt, build upon this work non-commercially, and license their derivative works on different terms, provided the original work is properly cited and the use is non-commercial. See: http://creativecommons.org/ licenses/by-nc/4.0/

(C) Article author(s) (or their employer(s) unless otherwise stated in the text of the article) 2017. All rights reserved. No commercial use is permitted unless otherwise expressly granted.

\section{REFERENCES}

1. Brierley J, Larcher V. Emergency research in children: options for ethical recruitment. J Med Ethics 2011;37:429-32.

2. U.S. Department of Health and Human Services: Food and Drug Administration. Exception from informed consent requirements for Emergency Research; guidance for Institutional Review Boards, Clinical Investigators, and Sponsors, 2013. cited May 2017. https:// http://www.fda.gov/downloads/regulatoryinformation/guidances/u cm249673.pdf.

3. Biros MH. Research without consent: exception from and waiver of informed consent in resuscitation research. Sci Eng Ethics 2007;13:361-9.

4. NHMRC. National Statement on Ethical Conduct in Human Research. NHaMR C, ed: Australian Govenrment, 2007.

5. World Medical Association. World Medical Association Declaration of Helsinki: ethical principles for medical research involving human subjects. JAMA 2013;310:2191-4.

6. Woolfall K, Frith L, Gamble C, et al. How parents and practitioners experience research without prior consent (deferred consent) for emergency research involving children with life threatening conditions: a mixed method study. BMJ Open 2015;5:e008522.

7. Gamble C, Nadel S, Snape D, et al. What parents of children who have received emergency care think about deferring consent in randomised trials of emergency treatments: postal survey. PLoS One 2012;7:e35982.

8. Maitland K, Molyneux S, Boga M, et al. Use of deferred consent for severely ill children in a multi-centre phase III trial. Trials 2011;12:90.

9. Molyneux S, Njue M, Boga M, et al. 'The words will pass with the blowing wind': staff and parent views of the deferred consent process, with prior assent, used in an emergency fluids trial in two African hospitals. PLoS One 2013;8:e54894.

10. Woolfall K, Young B, Frith L, et al. Doing challenging research studies in a patient-centred way: a qualitative study to inform a randomised controlled trial in the paediatric emergency care setting. BMJ Open 2014;4:e005045.

11. Bahora M, Sterk CE, Elifson KW. Understanding recreational ecstasy use in the United States: a qualitative inquiry. Int J Drug Policy 2009;20:62-9.

12. Vågan A. Medical students' perceptions of identity in communication skills training: a qualitative study. Med Educ 2009;43:254-9.

13. Birks M, Mills J. Grounded theory - a practical guide. Second Edition. London EC1Y 1SP: Sage Publications Inc, 2015.

14. Tong A, Sainsbury P, Craig J. Consolidated criteria for reporting qualitative research (COREQ): a 32-item checklist for interviews and focus groups. Int J Qual Health Care 2007;19:349-57.
15. Dalziel SR, Furyk J, Bonisch M, et al. A multicentre randomised controlled trial of levetiracetam versus phenytoin for convulsive status epilepticus in children (protocol): Convulsive Status Epilepticus Paediatric Trial (ConSEPT) - a PREDICT study. BMC Pediatr 2017;17:152. Accepted for publication.

16. Franklin D, Dalziel S, Schlapbach LJ, et al. Early high flow nasal cannula therapy in bronchiolitis, a prospective randomised control trial (protocol): A Paediatric Acute Respiratory Intervention Study (PARIS). BMC Pediatr 2015;15:183.

17. Patton MQ. Qualitative research \& evaluation methods. Third Edition ed. London: SAGE Publishing Ltd, 2015.

18. Biros MH, Sargent C, Miller K. Community attitudes towards emergency research and exception from informed consent. Resuscitation 2009;80:1382-

19. Booth MG, Lind A, Read E, et al. Public perception of emergency research: a questionnaire. Eur $J$ Anaesthesiol 2005;22:933-7.

20. Bulger EM, Schmidt TA, Cook AJ, et al. The random dialing survey as a tool for community consultation for research involving the emergency medicine exception from informed consent. Ann Emerg Med 2009;53:341-50.

21. Dickert NW, Mah VA, Baren JM, et al. Enrollment in research under exception from informed consent: the Patients' Experiences in Emergency Research (PEER) study. Resuscitation 2013;84:1416-21.

22. Goldstein JN, Espinola JA, Fisher J, et al. Public opinion of a stroke clinical trial using exception from informed consent. Int J Emerg Med 2010;3:385-9.

23. Kämäräinen $A$, Silfvast $T$, Saarinen $S$, et al. Conduct of emergency research in patients unable to give consent-experiences and perceptions of patients, their consent providing next of kin, and treating physicians following a prehospital resuscitation trial. Resuscitation 2012;83:81-5.

24. Morris MC, Nadkarni VM, Ward FR, et al. Exception from informed consent for pediatric resuscitation research: community consultation for a trial of brain cooling after in-hospital cardiac arrest. Pediatrics 2004;114:776-81.

25. Sims CA, Isserman JA, Holena D, et al. Exception from informed consent for emergency research: consulting the trauma community. $J$ Trauma Acute Care Surg 2013;74:157-65. discussion 65-6.

26. Declaration of Helsinki: World Medical Association. 2013 [cited Jan 12 2017]. Available from: http://www.wma.net/en/20activities/ 10ethics/10helsinki/.

27. Maitland K, Kiguli S, Opoka RO, et al. Mortality after fluid bolus in African children with severe infection. N Engl J Med 2011;364:2483-95.

28. Appelbaum PS. Clarifying the ethics of clinical research: a path toward avoiding the therapeutic misconception. Am J Bioeth 2002;2:22-3.

29. Morrow BM, Argent AC, Kling S. Informed consent in paediatric critical care research-a South African perspective. BMC Med Ethics 2015;16:62.

30. Harron K, Woolfall K, Dwan K, et al. Deferred consent for randomized controlled trials in emergency care settings. Pediatrics 2015;136:e13 16-e1322.

31. Salzman JG, Frascone RJ, Godding BK, et al. Implementing emergency research requiring exception from informed consent, community consultation, and public disclosure. Ann Emerg Med 2007;50:448-55. 